\title{
Photodetachment in cold ion traps
}

\section{Rotational state selective $\mathrm{C}_{2} \mathrm{H}^{-}$losses from quantum dynamics}

\author{
Barry P. Mant ${ }^{1}$, Markus Nötzold ${ }^{1}$, Lola González-Sánchez ${ }^{2}$, Roland Wester ${ }^{1}$, and Franco A. Gianturco ${ }^{1, a}$ \\ 1 Institut für Ionenphysik und Angewandte Physik, Universität Innsbruck, Technikerstr. 25, 6020 Innsbruck, Austria \\ 2 Departamento de Química Física, University of Salamanca, Plaza de los Caídos sn, 37008 Salamanca, Spain
}

Received 21 January 2020 / Received in final form 21 April 2020

Published online 25 June 2020

(C) The Author(s) 2020. This article is published with open access at Springerlink.com

\begin{abstract}
We present a computational investigation of the photodetachment (PD) dynamics of $\mathrm{C}_{2} \mathrm{H}^{-}$in its ground, electronic ${ }^{1} \Sigma$ state, cooled in an ion trap with He as a buffer gas. Our analysis employs a range of $\mathrm{PD}$ rates from quantum dynamics, selective choices of the anion's initial rotational state populations as the laser is switched on, and different operational trap conditions. Our results show that a variety of outcomes for the initial state-dependent PD process is possible by selecting different initial conditions in the cold trap, the latter generating a dynamical interplay between the anion's populations of rotational states, induced by the buffer gas, and the chosen laser frequency and laser power. Specific parameter selections during the PD process will be shown to cause different abundances of the rotational states of the anion in the trap.
\end{abstract}

\section{Introduction}

A detailed understanding of the physics of electronmolecule and photon-molecule collision dynamics is of fundamental importance for the general field of molecular physics and it is indeed needed in various applications such as the manufacturing of semiconductor devices, plasmadriven chemical synthesis and in the modelling of atmospheric chemistry and physics [1-4]. It is also an important tool for investigating selective-state chemistry within the low temperature conditions of cold ion traps and, in the case of photon-induced processes, for clarifying the role of photodetachement pathways in producing assemblies of molecular anions with a specified internal ro-vibrational state $[5-7]$.

Theoretical and computational studies play an important role both for understanding the underlying dynamics of these processes and because they can provide quantitative predictions of the appropriate data. In the case of processes involving ions and occurring in a cold trap, where the presence of a buffer gas (usually He atoms) controls the initial populations of the internal states of the molecular ions, one needs to combine knowledge of the scattering in the gas phase at the selected trap temperatures with the modeling of the process where laser light

\footnotetext{
* Contribution to the Topical Issue "Atomic Cluster Collisions (2019)", edited by Alexey Verkhovtsev, Pablo de Vera, Nigel J. Mason, Andrey V. Solov'yov.

a e-mail: francesco.gianturco@uibk.ac.at
}

interacts with the molecular anions leading to the ejection of a single electron. In other words, one needs to combine knowledge of the intermolecular forces for the anion-buffer gas interactions with modeling of the kinetics of the photodetachment (PD) processes (e.g. see references [8] and [9]).

The dynamics of PD near threshold, i.e. when the laser wavelength matches the positive Electron Affinity (EA) of the molecular ion taken to be in a specific internal ro-vibrational state, can be well approximated by making use of Wigner's Law [10], which is often employed to extrapolate the size and behaviour of experimental PD cross sections to energy threshold in the determination of experimental electron affinities. Furthermore, photodetachment of molecular anions is more complicated than for atomic PD processes due to the increasing number of accessible pathways. Molecules exhibit a higher density of lower-lying electronic states while one must further consider the increase in the density of states due to the additional nuclear motion, i.e. ro-vibrational and predissociative states of the initial anion as the bound electron is moved into the continuum [11] by the laser beam.

$\mathrm{PD}$ of negative ions also provides an important tool for the studies of reaction dynamics where one wants to employ the initial anionic partner to be specifically produced in a given ro-vibrational state of its ground electronic state $[12,13]$. In the present report we shall present a computational study of a variety of the operating conditions in cold ion traps with the aim of producing the photo-detaching species in some prevalent rotational state of its ground vibrational and electronic state. In particular, we shall consider the case of trapping the $\mathrm{C}_{2} \mathrm{H}^{-}$anion 
in its ground vibrational state, but in a variety of its lower rotational states, via collisional cooling by the uploaded buffer gas. The latter will be He in the present example, although earlier on we have also considered the case of $\mathrm{Rb}$ prepared as a cold partner by sympathetic cooling [7].

There are various reasons why we have decided to work in detail on simulating the PD process for the present system. First of all, actual experiments in cold traps involving this very molecule are to be planned in our laboratory and therefore it would be useful to provide in advance realistic indicators of the state-selective PD features one expects to observe. The title molecule, as we shall show below, is further able to have a rather extended number of rotational states to be populated in the cold trap after equilibration with the buffer gas, a feature that will help in showing how specific operating conditions can induce state-selectivity in their population after the PD process and therefore provide the anions in a rotational state other than the ground state. Furthermore, the $a b$ initio evaluation of the interaction forces between this molecule and the buffer gas has already been done and is available in the literature [14]. We have already employed that specific potential energy surface (PES) to carry out scattering calculations for this system in order to compare its state-changing rotational dynamics with other similar anions [15] so that all the relevant cross sections and rates are already available for us to use in the present extension to a more detailed description of the trap evolution. The following section will therefore report, briefly, the general features of the known interaction forces, while Section 3 will outline the formulation of the PD cross section behaviour near threshold and the expected relative strengths of transition moments from specific initial rotational levels of the target anion. We shall then discuss in Section 4 the details of the quantum kinetics with different operating conditions and will then present our results. Our conclusions will be reported in Section 5.

\section{Interaction forces and anion's rotational spacing}

A convenient representation of the interaction forces between the partners is often given in terms of multipolar radial coefficients from a standard expansion in Legendre polynomials:

$$
V\left(r_{\text {eq }} \mid R, \theta\right)=\sum_{\lambda}^{\lambda_{\max }} V_{\lambda}\left(r_{\text {eq }} \mid R\right) P_{\lambda}(\cos \theta)
$$

for the case of the molecule described as a Rigid Rotor $(\mathrm{RR})$, the $V_{\lambda}\left(r_{\text {eq }} \mid R\right)$ terms provide a measure of the relative strength and radial extension of the angular "torque" applied during the interaction by the impinging atom to the rotating molecule. In qualitative, classical terms, the PES allows pictorially to see how much, at any given radial distance, the angular interaction can change, thereby affecting the dynamics of state-changing collisions by specifically coupling different rotational states within the Coupled Channel (CC) formulation of the quantum
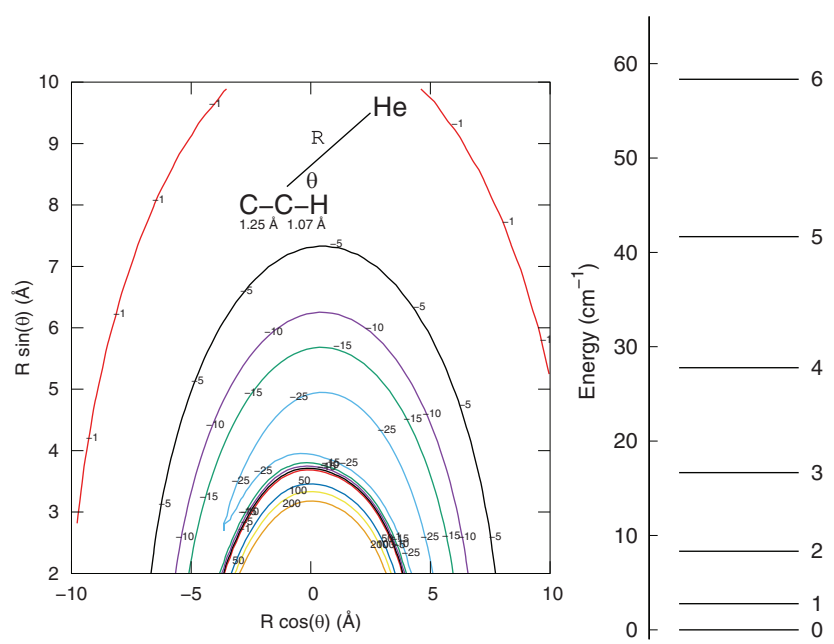

Fig. 1. The left panel shows a $2 \mathrm{D}$ representation of the present PES from reference [14]. The molecule is located on the horizontal axis with the origin at the center-of-mass ( $\mathrm{H}$ atom on the positive side). Distances are given in angle-scaled Angström and the energy levels in $\mathrm{cm}^{-1}$. The bond lengths for the linear $\mathrm{C}_{2} \mathrm{H}^{-}$molecule [16] and coordinate definitions are also shown as an inset. The right panel shows the rotational spacing between the lower levels of the RR target, also given in units of $\mathrm{cm}^{-1}$. See main text for further details.

dynamics, as further discussed below. The details of that potential energy surface (PES) have been already published a while ago [14] and have been discussed again by us in another publication [15]. We will therefore refer to those two publications for fuller details. We present in the panels of Figure 1 some of the properties of the PES of the present work.

The 2D mapping of the potential clearly shows the asymmetric location of the weak attractive well: off-center and on the direction of the $\mathrm{C}-\mathrm{H}$ bond. The multipolar coefficients, already discussed in our earlier work [15], represent its anisotropic features and indicate the range of action of the PES during rotationally state-changing dynamics is directly coupling transitions with $\Delta j=1$ and $\Delta j=2$ between the populated rotational states in the cold trap, as we have already discussed in some detail in that reference and will be further discussed below. The right panel additionally shows the relative energy spacing between the lower rotational levels which can be excited by collisions for energies up to about $60 \mathrm{~cm}^{-1}$.

In our previous study [15] it was shown that at temperatures up to about $50 \mathrm{~K}$, the first 7 or 8 rotational levels of the present anion were likely to be populated during the rapid collisional equilibration inside the cold trap. Hence, the rotational level spacing reported in Figure 1 indicates that excitations up to $j=6$ can be reached from the available relative energy of about $100 \mathrm{~cm}^{-1}$ during the initial setting of the ion in the trap: this aspect, in relation to the PD process, will be further discussed in the sections below. In particular, we will analyze in the next section how to estimate the relative sizes of the rotational statedependent PD cross sections from the relevant angular momentum coupling schemes for the present system. 


\section{Photodetachment cross sections and rates}

The photodetachment cross section $\sigma_{\mathrm{PD}}$, as a function of the photon frequency $\nu$, for a transition from a initial state $|i\rangle$ to a final continuum state $|f\rangle$ can be expressed as [17]

$$
\sigma_{\mathrm{PD}}(\nu)=\frac{4 \pi^{2} \nu}{c}|\langle f|\vec{\epsilon} \cdot \vec{d}| i\rangle|^{2}
$$

where $\vec{\epsilon}$ is the polarisation vector of the photon and $\vec{d}$ is the dipole operator. For a diatomic molecule (or linear rigid rotor) within the Born-Oppenheimer approximation, the initial and final states can be written as

$$
\begin{aligned}
& |i\rangle=\sqrt{\frac{2 J^{\prime \prime}+1}{8 \pi^{2}}} D_{m^{\prime \prime}, 0}^{J^{\prime \prime} *}(\alpha, \beta, \gamma) \chi^{\prime \prime} \phi^{\prime \prime} \\
& |f\rangle=\sqrt{\frac{2 J^{\prime}+1}{8 \pi^{2}}} D_{m^{\prime}, 0}^{J^{\prime} *}(\alpha, \beta, \gamma) \chi^{\prime} \phi^{\prime}
\end{aligned}
$$

where $D_{m, 0}^{J *}(\alpha, \beta, \gamma)$ is a Wigner D function with the Euler angles as arguments and $J$ and $m$ are the total angular momentum and projection along the z-axis respectively. $\chi$ are eigenfunctions of the vibrational coordinate and $\phi$ are eigenfunctions of the electronic coordinates.

The electronic dipole transition term in equation (2) is proportional, near threshold, to a Wigner-type power law [10] that is however modified by the presence of the molecular dipole [18]. As a consequence the cross section now becomes proportional to $\left(E-E_{\text {th }}\right)^{p}$ where $E$ is the photon energy and $E_{\text {th }}$ is the threshold energy given as

$$
E_{\mathrm{th}}=E_{\mathrm{EA}}+B^{\prime} J^{\prime}\left(J^{\prime}+1\right)-B^{\prime \prime} J^{\prime \prime}\left(J^{\prime \prime}+1\right)
$$

where $E_{\mathrm{EA}}$ is the electron affinity and $B^{\prime}$ and $B^{\prime \prime}$ are the rotational constants of the neutral and anionic molecules respectively. The additional exponent $p$ now depends on the dipole of the neutral molecule and on the projection of the angular momentum onto the molecular axis [18]. It is essentially the new, system-specific factor which is altering the simpler Wigner-type power law through the molecular features of the target undergoing the PD process. It is well known that the parameter $p$ depends on the angular momentum $l$ of outgoing photoelectrons. Hence, above the threshold, $p=0.5$ for $s$-wave photo-detachment, and $p=1.5$ for $p$-wave photo-detachment. The work by Jia Zhou et al. [19] has shown that the photoelectron angular distributions of the channel from the ground-state $\mathrm{C}_{2} \mathrm{H}^{-}$ to the ground-state $\mathrm{C}_{2} \mathrm{H}$ has $\mathrm{p}$-wave characteristics and also that the different exponents' values provide at threshold marked differences in size between an $s$-wave dominated and $p$-wave dominated cross section. This is also consistent with the fact that the highest occupied molecular orbital of $\mathrm{C}_{2} \mathrm{H}^{-}$is an $s$-type molecular orbital. We shall discuss further below how sensitive the final, estimated $\sigma_{\mathrm{PD}}$ will be to different choices for the $p$ parameter discussed before. Such "sensitivity tests" will help us to better understand how significant the initial choice of a $p$-wave nature of the ejected photo-electron would be to our model.

The PD curve, representing the total cross section as a function of the laser frequency and for a collection of molecules at thermal equilibrium at the chosen temperature $T$, has been derived in detail elsewhere [20] so we shall not repeat it here but only give a brief outline.

In the actual trap experiments, as we shall further discuss below, we can control and select the population of the initial anion's rotational states by regulating the operating temperature of the buffer gas against which the molecular rotational states are thermalised by collision. Since they reach thermal equilibrium in the trap before the switching on of the PD laser (see next section), we do not need to explicitly include the Boltzmann term in equation (6). Thus, for a specifically selected initial rotational state $J^{\prime \prime}$ of the molecular anion the experimental PD cross section turns out to be proportional to the following expression:

$$
\sigma_{J^{\prime \prime}}^{\mathrm{PD}}(\nu) \propto \sum_{J^{\prime}=0}^{J_{\max }}\left|C_{J^{\prime \prime}, 0,1,0}^{J^{\prime}, 0}\right|^{2}\left(E-E_{\mathrm{th}}\right)^{p} \Theta\left(E-E_{\mathrm{th}}\right) .
$$

The Clebsch-Gordan coefficient given in equation (6) enforces the selection rule $\Delta J^{\prime \prime}= \pm 1$ for the specific PD process under consideration. Since the rotational levels' relative populations initially also sum to one, they have limited effect on the relative sizes of the state-dependent $\sigma_{J^{\prime \prime}}^{\mathrm{PD}}(\nu)$. Thus, to model the PD cross sections and the PD curve of above, the most important factors are the selected value for the $p$ parameter and the actual wavelength $\nu$ of the laser source with respect to the threshold of the specific electron-detachment process.

To make now a direct comparison with the experimental kinetics in the trap, the estimated values of $\sigma_{J^{\prime \prime}}^{\mathrm{PD}}(\nu)$ can be employed to model the corresponding $\mathrm{PD}$ rates by introducing an arbitrary parameter $\alpha(\nu)$ as done by us in our previous work [21] which provides a way of linking the estimated cross sections to the scalable $\mathrm{PD}$ rates given by

$$
K_{J^{\prime \prime}}^{P D}=\alpha(\nu) \sigma_{J^{\prime \prime}}^{\mathrm{PD}}(\nu)
$$

The scaling parameter is meant to include the relative role of the laser-driven features like laser-flux, spatial overlap, etc. with respect to the pure collisional rates that re-populate the anion's rotational levels via its interaction with the buffer gas. This specific aspect of the model will allow us to simulate either a "collision-dominated" situation or a "PD-dominated" situation where the laser strength is increased, as will be further discussed in the next section.

We are now in a position to obtain specific relative values of the rotational state-dependent PD cross sections for different choices of the parameter $p$, and for different selections of the laser frequency with respect to the experimental threshold given by the EA value of the anion $\mathrm{C}_{2} \mathrm{H}^{-}$. We report in Tables 1 and 2 the values of $\sigma_{J^{\prime \prime}}^{\mathrm{PD}}(\nu)$ computed using equation (6), relative to the largest value obtained from that equation. Our results show how the relative size of the cross sections change as a function of the $p$ exponent and for the two choices of photon frequencies we are listing as examples. The rotational constants for $\mathrm{C}_{2} \mathrm{H}^{-}$ and $\mathrm{C}_{2} \mathrm{H}$ which are 1.39 and $1.45 \mathrm{~cm}^{-1}$ respectively $[22,23]$ and the electron affinity is $2.9689 \mathrm{eV}$ [19]. The values of $p$ are chosen within the range often obtained in experimental fittings of the observed PD curves, as shall be further 
Table 1. Relative values of $\sigma_{J^{\prime \prime}}^{\mathrm{PD}}(\nu)$ as a function of $p$ for a value of $\nu=$ electron affinity $(\mathrm{EA})$ of: $23945 \mathrm{~cm}^{-1}(2.9689 \mathrm{eV})$.

\begin{tabular}{cccccccccccc}
\hline$J^{\prime \prime}$ & 0.5 & 0.6 & 0.7 & 0.8 & 0.9 & 1.0 & 1.1 & 1.2 & 1.3 & 1.4 & 1.5 \\
\hline & & & & & & & & & & & \\
0 & 0.00 & 0.00 & 0.00 & 0.00 & 0.00 & 0.00 & 0.00 & 0.00 & 0.00 & 0.00 & 0.00 \\
1 & 0.31 & 0.27 & 0.23 & 0.19 & 0.16 & 0.14 & 0.12 & 0.10 & 0.08 & 0.07 & 0.06 \\
2 & 0.51 & 0.46 & 0.41 & 0.37 & 0.33 & 0.30 & 0.27 & 0.24 & 0.22 & 0.20 & 0.18 \\
3 & 0.65 & 0.60 & 0.56 & 0.52 & 0.49 & 0.45 & 0.42 & 0.39 & 0.37 & 0.34 & 0.32 \\
4 & 0.76 & 0.72 & 0.69 & 0.66 & 0.63 & 0.60 & 0.57 & 0.55 & 0.53 & 0.50 & 0.48 \\
5 & 0.85 & 0.83 & 0.81 & 0.78 & 0.76 & 0.74 & 0.72 & 0.70 & 0.69 & 0.67 & 0.65 \\
6 & 0.93 & 0.92 & 0.91 & 0.90 & 0.88 & 0.88 & 0.87 & 0.85 & 0.84 & 0.83 & 0.82 \\
7 & 1.00 & 1.00 & 1.00 & 1.00 & 1.00 & 1.00 & 1.00 & 1.00 & 1.00 & 1.00 & 1.00 \\
\hline
\end{tabular}

Table 2. Relative values of $\sigma_{J^{\prime \prime}}^{\mathrm{PD}}(\nu)$ as a function of $p$ for $\nu=$ $E A+50 \mathrm{~cm}^{-1}$.

\begin{tabular}{ccccccc}
\hline$J^{\prime \prime}$ & 0.5 & 0.7 & 0.9 & 1.1 & 1.3 & 1.5 \\
\hline 0 & 1.000 & 1.000 & 1.000 & 1.000 & 1.000 & 1.000 \\
1 & 0.997 & 0.997 & 0.997 & 0.997 & 0.997 & 0.999 \\
2 & 0.993 & 0.992 & 0.991 & 0.992 & 0.993 & 0.996 \\
3 & 0.986 & 0.983 & 0.983 & 0.984 & 0.987 & 0.991 \\
4 & 0.976 & 0.972 & 0.971 & 0.973 & 0.978 & 0.986 \\
5 & 0.963 & 0.957 & 0.956 & 0.959 & 0.967 & 0.979 \\
6 & 0.948 & 0.940 & 0.938 & 0.944 & 0.955 & 0.972 \\
7 & 0.928 & 0.918 & 0.917 & 0.925 & 0.941 & 0.965 \\
\hline
\end{tabular}

discussed below. The highest value shown in the tables corresponds to the theoretical value of the parameter $p$ already discussed above.

From Table 1 the higher $J^{\prime \prime}$ states yield higher relative values of $\sigma_{J^{\prime \prime}}^{\mathrm{PD}}(\nu)$ at threshold $(\nu=\mathrm{EA})$ because of the $\left(E-E_{\text {th }}\right)$ factor in equation (6). The $J^{\prime \prime}=0$ state does not photodetach as $\nu=h f=E=E_{\text {th }}$ so $\sigma_{J^{\prime \prime}}^{\mathrm{PD}}(\nu)=0$. From the data in the Tables we see that there is a visible variation of the relative $\sigma_{\mathrm{PD}}$ values, for each chosen $p$ exponent, along the range of rotational quantum numbers of the populated anion's state with the larger values of $p$ giving a larger spread of relative sizes for $\sigma_{J^{\prime \prime}}^{\mathrm{PD}}(\nu)$. From Table 2 we further see that the $\sigma_{J^{\prime \prime}}^{\mathrm{PD}}(\nu)$ are fairly similar in size along the sequence of $J^{\prime \prime}$ values when the chosen photon energies are well above the threshold: changing $p$ makes now little difference to the relative sizes of the cross sections, a feature that will be significant in the discussion of the following Section, where a range of parameters will be employed to model the experiments. On the other hand, we see from the previous Table 1 that for photon frequencies close to the EA value the estimated $\sigma_{\mathrm{PD}}$ change more as $p$ is changed, indicating again the additional role of the relative residual energy of the ejected, photo-detached electron in conditions above the threshold energy.

\section{Modelling PD selectivity by changing trap conditions}

The process which we wish to analyse involves the following steps: $(i)$ collisional re-distribution among the populated rotational levels of the anion by the interaction with He buffer gas at the trap temperature; (ii) switching on the photo-detaching laser after rotational population equilibration and varying its wavelength to selectively depopulate different rotational states of the anion; (iii) change other operating conditions such as He buffer gas density, trap temperature and laser power/wavelength in order to find the best parameter choice for the present system. We need to know beforehand the state-changing collisional rates for the molecular anion in the trap at different operating temperatures and the kinetics of the evolution of the rotational state populations during collisional equilibration, besides selecting values of the PD rates obtained from the above cross sections as functions of the initial rotational state. Such quantities have been already calculated before by us [15] and therefore we will not repeat here the computational procedures. As a comment about the calculated cross sections and rates for state-to-state inelastic processes suffices it to say that the dominant inelastic processes we found for this system are those for which the state-changing values of $\Delta J^{\prime \prime}= \pm 1,2$ and for which the rates at a temperature of $15 \mathrm{~K}$ are of the order of $1.5 \times 10^{-10}$ in units of $\mathrm{cm}^{3} \mathrm{~s}^{-1}$. As a comparison, the corresponding Einstein spontaneous emission coefficients are about from $10^{-3}$ to $10^{-5}$ smaller than the collisional rates cited above for this system [15]. The computational method for the present quantum dynamics calculations will be further mentioned below.

\subsection{The kinetics scheme in the trap}

Once the state-to-state inelastic integral cross sections are known, the rotationally inelastic rate constants $k_{j \rightarrow j^{\prime}}(T)$ can be evaluated as the convolution of the cross sections over a Boltzmann distribution of the relative collision energies. In equation (8):

$$
k_{j \rightarrow j^{\prime}}(T)=\left(\frac{8}{\pi \mu k_{B}^{3} T^{3}}\right)^{1 / 2} \int_{0}^{\infty} E \sigma_{j \rightarrow j^{\prime}}(E) e^{-E / k_{B} T} d E .
$$

The above rates were therefore obtained from a direct evaluation of the state-changing rotationally inelastic cross sections using a multichannel, Coupled-Channel (CC) quantum treatment of the dynamics driven by the PES briefly described before and pictorially shown in Figure 1. The computational procedure involved the use of our in-house code ASPIN previously described many 
times in the literature: e.g. see references [24,25], thus we will not repeat here its computational details.

To further continue modelling the rotational population time evolution dynamics, the master equations are solved using the collisional thermal rates at each chosen trap temperature and selected He density:

$$
\frac{d n_{i}(t)}{d t}=\sum_{j \neq i} n_{j}(t) C_{j i}(T)-n_{i}(t) \sum_{i \neq j} P_{i j}(T) .
$$

Here the $P_{i j}(T)$ are the rates for the destruction of level $i$, while its formation rates are given by the $C_{j i}(T)$ coefficients. During the collisional step, i.e. before the laser is switched on, the coefficients are given as:

$$
\begin{aligned}
P_{i j}(T) & =\eta_{\mathrm{He}} k_{i \rightarrow j}(T) \\
C_{j i}(T) & =\eta_{\mathrm{He}} k_{j \rightarrow i}(T) .
\end{aligned}
$$

This describes the "collision-driven" time evolution process of thermalising the relative population of the rotational levels of the anion at the selected buffer gas temperature for a given density $\eta_{\mathrm{He}}$ in the trap. Once the thermalisation process is reached and the PD laser is turned on, one needs to modify the master equations by including the PD rates discussed in the previous section and linked to the PD cross sections:

$$
\frac{d n_{i}(t)}{d t}=\sum_{j \neq i} n_{j}(t) C_{j i}(T)-n_{i}(t)\left(\sum_{i \neq j} P_{i j}(T)+K_{i}^{P D}\right)
$$

where $K_{i}^{\mathrm{PD}}$ is the additional destruction rate of the selected level $i$ caused by the PD laser. The set of rates $K_{i}^{\mathrm{PD}}$ is critical in the experiment and for the numerical simulation because they drive the destruction of both the population of one specific rotational level $i$ and of all the molecular ions which have been populating that specific state. In the experiments of this study these rates depend on the laser photon flux and on the overlap between the laser beam and the ion cloud within the trap. Since these parameters, as well as the absolute values of the state-tostate PD cross sections, are presently unknown for the title molecular anion, we have introduced a scaling parameter according to the relation given by equation (7). If one now employs the relative values of the corresponding $\sigma_{J^{\prime \prime}}^{\mathrm{PD}}(\nu)$ as a function of the exponent $p$ defined in equation (6), and further selects the specific energetics for the laser wavelength presented in Tables 1 and 2, it becomes possible to test the modelling using different values of the PD cross sections needed in equation (7). The specific results we have obtained from the present calculations will be discussed in the following subsection.

\subsection{Results from present calculations}

An example of such calculations is reported by the panels shown in Figure 2. The figure presents in its four panels the computed fractional evolutions of the rotational state population of the anion under different choices of the cold trap's parameters. The rotational populations at $t=0$
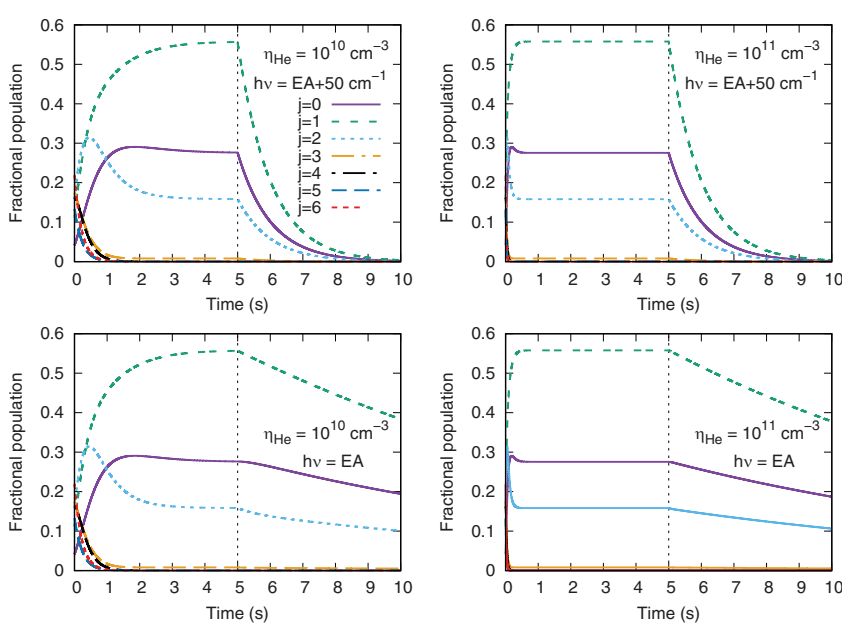

Fig. 2. Different selections of the trap parameters during the $\mathrm{PD}$ kinetics and corresponding evolution of anionic fractional populations. Buffer gas densities and laser wavelengths are reported in each panel. The trap temperature is set to $5 \mathrm{~K}$ in all panels. The parameter $p$ is chosen to be 1.5. See main text for further details.

are chosen as those at $50 \mathrm{~K}$. The lower two panels select the laser wavelength equal to the EA value of the anion in its $J^{\prime \prime}=0$ rotational state, while the buffer gas densities vary by one order of magnitude from left to right panels. The two upper panels show the same process but employing instead a laser wavelength which delivers an energy above the EA threshold by $50 \mathrm{~cm}^{-1}$, as shown in the earlier tables reporting the PD cross sections.

It is interesting to note the following from the evolutionary kinetics reported in Figure 2:

$(i)$ if one looks at the rotational state populations during the stage preceding the laser action (left parts in all four panels) we see the purely collisional step of repopulating the relevant states of the molecular anion under the driving action of the buffer gas. Hence, when going from the left to the right panel we see that the increase of the buffer gas density makes the thermalisation to the gas temperature (in these examples: $5 \mathrm{~K}$ ) of the molecular population faster: in the left-side panels this is achieved within about $1-2$ sec., while in the panels on the right less just about $0.1 \mathrm{sec}$. is sufficient to bring the rotational state populations to equilibrate with the $5 \mathrm{~K}$ temperature of the trap;

(ii) as given in the previous sections, equation (6) indicates that the PD cross section increases from threshold through an exponential factor in relation to the specific system and operating conditions, by fitting the experimental PD function through the modified Wigner-type law. Since no experiments are as yet available for the title molecular anion, in our present modelling we have chosen the exponent $p$ to vary over a range of values which have been obtained and employed by the experimental fittings of earlier measured cross sections on similar systems $[9,21]$. Table 1 therefore indicates that values of $p$ from 0.5 to 1.5 provide relative values of the $\mathrm{PD}$ cross sections which differ from each other as $J^{\prime \prime}$ changes [9]. The model 

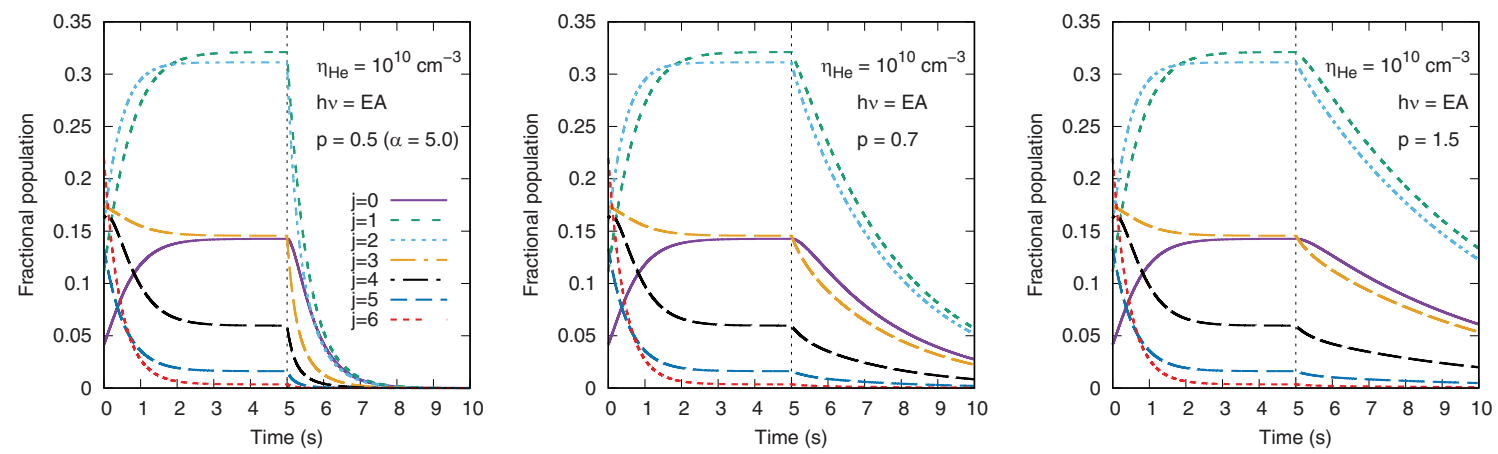

Fig. 3. Computed kinetics behaviour in the cold trap under different conditions from those of Figure 2. The trap temperature is here $15 \mathrm{~K}$, while all other parameters are explicitly reported in each panel. See main text for further details.

calculations of Figure 2 have therefore been obtained using a test value of $p=1.5$, and the different cross section relative values of Tables 1 and 2 . The scaling value employed to get the rates from equation (7) was taken to be $\alpha=1.0$ in Figure 2. In other words, our present model calculations acknowledge the presence of a $p$-wave dominant electron emission and vary both gas density and PD laser wavelength in the panels of this figure;

(iii) if we now compare the loss rates reported in the right-hand side of all panels after the laser is switched on, we see clearly that when the chosen wavelength matches the EA value (two lower panels) the laser produces decay rates which are slower than when the energy of the laser source is increased above the EA threshold by a test value of $50 \mathrm{~cm}^{-1}$. Thus, the upper panels of Figure 2 show now much faster loss rates than in the calculations in the lower panels;

(iv) as in previous comments, we further note here that, after the laser is switched on, the populations of the anion's rotational states at $5 \mathrm{~K}$ are larger for molecules in the $j=1$ state than those in $j=0$ and $j=2$, while higher levels are negligibly present within the equilibrated rotational population in the cold trap. The relative populations of the anion's rotational levels under steadystate conditions are given by Figure 6 and indicate around $5 \mathrm{~K}$ the same population differences seen in Figure 2. We should also note here that the PD rates chosen are describing collision-dominated operating conditions in the trap, thus explaining the similarity of behaviour.

Operating conditions have been further changed for the results in Figure 3: to underline the importance of the actual strength of the $K_{J^{\prime \prime}}^{\mathrm{PD}}$ selected in the experiments. The following considerations can be had by looking at those results:

( $i$ ) the trap temperature has been increased to $15 \mathrm{~K}$, to model the more usually achieved trap temperatures in experiments [7]. We see from these data that the collisional thermalisation step on the left-hand-side of all panels, includes now a larger number of rotational states with significant population: $J^{\prime \prime}=1$ and $J^{\prime \prime}=2$ are by and large the top two levels in terms of relative abundance, while the $J^{\prime \prime}=3$ level shows the same abundance as the ground-state rotational state. This is due to the features of the stationary population of rotational levels as shown in Figure 6. We further see different changing rates of the populations from the initial loading of the ion in the trap to reach the steady-state populations at the trap temperature. This is due to the different relative size of the state-changing rates which drive the equilibration process;

(ii) the loss rates in Figure 3 after the laser is switched on, report in the three panels the changes when the exponent $p$ is increased from 0.5 up to 1.5. Although we know that the physically realistic choice for the $p$ value is that of a dominant $p$-wave for the ejected electron [19], it is still interesting to gauge the model sensitivity to modifications of that parameter;

(iii) we see in the data from the center and right panels in that figure, where the $\alpha$ parameter of equation (7) has been kept to the same value of 1.0, that the loss rates decrease more slowly when the exponent increases from the left to the right panel. For the largest $p$ value selected, the one expected to be the closest to the theoretically suggested value, the losses of the two highest populated levels after $10 \mathrm{~s}$ are more than twice smaller than for the $p=0.7$ value of the central panel. This is in keeping with the results shown in the previous tables, where the relative PD cross section values are seen to decrease, for the same rotational state, as the parameter $p$ is increased;

$(i v)$ in the left panel of the same figure the factor $\alpha(\nu)$ employed for scaling the PD rates has now been increased to 5.0 , while the parameter $p$ has been kept at 0.5 . We now see that the loss rates are much faster for all the levels. We are not any more under "collision dominated" conditions as in the previous panels and therefore the strength of the laser is now driving the decay rates from the various levels more than the state-dependent collisional repopulation step. These data also show that the threshold law employed for the ejected photo-electron is becoming less significant as we leave the "collision- dominated" regime and move to a "photon-dominated" regime where a more powerful laser would mask the differences among collision rates in driving the anion's rotational state populations once switching the laser on. The role played by differences between PD cross sections shown by our model in the columns of Table 1 is also reduced by the increased laser power so that the populations of all levels in the trap decay faster and more similarly to each other. 

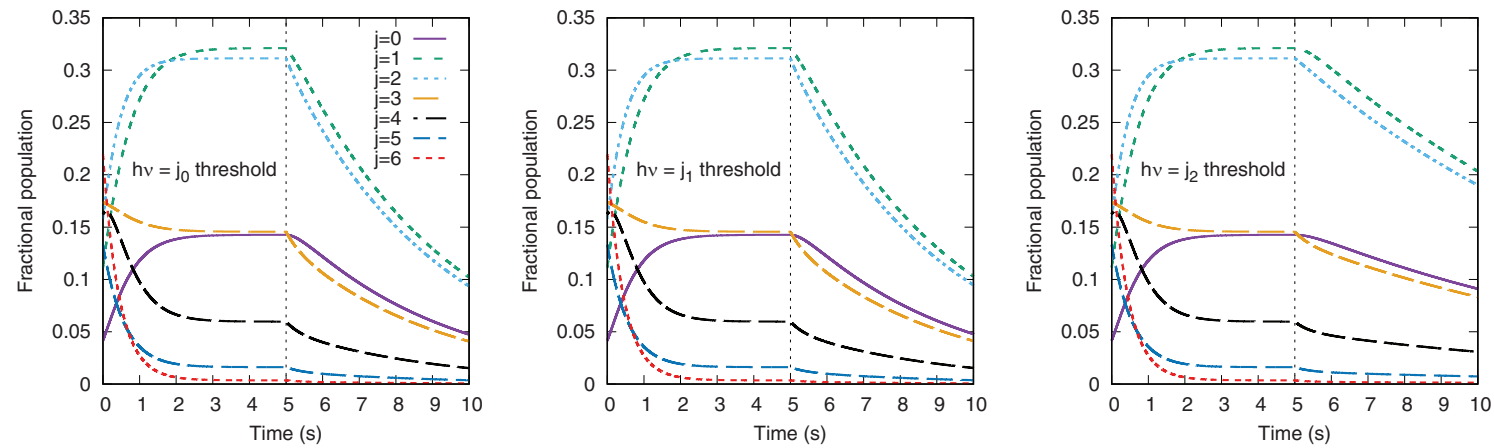

Fig. 4. Computed PD kinetics by selectively photo-detaching the electron from anions in different rotational states. See main text for further details. In all the panels the trap temperature is $15 \mathrm{~K}$ and the buffer gas density is $10^{10} \mathrm{~cm}^{-3}$. The $K_{J^{\prime \prime}}^{\mathrm{PD}}$ values are selected for the exponential parameter $p=1.5$ (see Tab. 1) while the $\alpha(\nu)$ parameter of equation (7) is set to 1.0 .
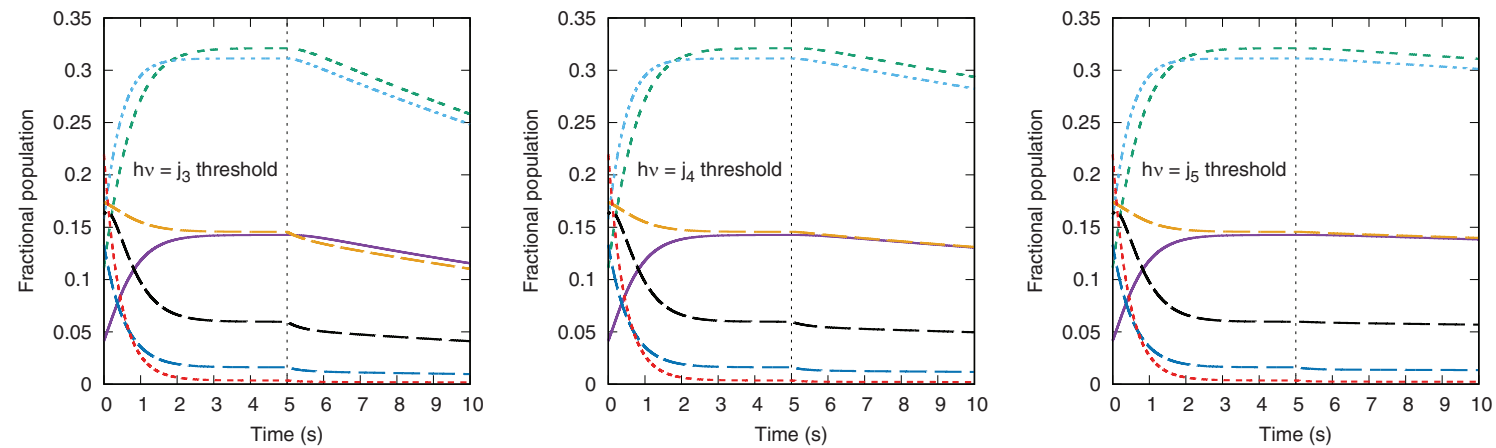

Fig. 5. Same type of calculations as those reported by Figure 4, but in this figure using different threshold rotational levels for the PD laser frequency. The key for the states is the same as in earlier figures. See main text for further details.

In conclusion, the results from a variety of parameters of the trap operation indicate here that changes in buffer gas densities alter the speed at which the anion's rotational states achieve equilibration at the chosen trap temperature, but have largely no effect on the ensuing kinetics of the PD step (laser power on in the trap) when the interplay between collisional re-equilibration and laser strength controls those decay rates. The differences in the collisional state-changing rates alter state populations during the detachment step by collisional re-equilibration between the populations of different levels. The differences are however reduced as one moves to the "photondominated" regimes where the laser power is increased (i.e. the individual $K_{J^{\prime \prime}}^{P D}$ values are larger) and therefore the relative differences between state-dependent PD cross sections (see Tabs. 1 and 2) become less significant, showing similarly rapid losses largely independent of the $p$ choice (see left panel in Fig. 2). Further aspects of the PD process in the present system will be shown and discussed in the following section.

\subsection{Possible selectivity effects from laser frequency changes}

The calculations reported by Figures 4 and 5 change the PD step in that they introduce a form of state-selectivity from the chosen wavelength of the electron-detaching laser. In other words, different rotational levels (see right-hand panel in Fig. 1) of the trapped anion are used as starting reference energy for the laser, so that for the case of, say, " $j_{2}$ threshold" the $K_{J "}^{\mathrm{PD}}$ values for $J^{\prime \prime}=0$ and $J^{\prime \prime}=1$ are taken to be zero and the electron-detaching process starts with a lower laser frequency than that required by matching the EA value at the $J^{\prime \prime}=0$ initial level of the anion. This model requires a re-scaling of the PD rates, following the procedure outlined in the previous section, to the largest one of the remaining $\mathrm{PD}$ rates which are not set to zero in this trap condition. The $p$ exponent has been chosen to be that of a dominant $p$-wave electron emission. The $\alpha$ parameter of equation (7) has been chosen as 1.0 in the experimental modelling of Figures 4 and 5, where all levels above the stated thresholds are undergoing the PD process.

The differences in behaviour between the loss rates reported in three panels of Figure 4 indicate the dynamical interplay between collisional re-populations of the rotational levels of the anion in the trap and the loss rates induced by the laser power features, as outlined before. In all the calculations the trap temperature has been fixed to $15 \mathrm{~K}$ in order to bring more rotational states into play. The buffer gas density has also been fixed at the lower value of $10^{10} \mathrm{~cm}^{-3}$. The operating conditions also reflect collision-dominated situations by fixing the $\alpha(\nu)$ parameter to 1.0 .

When the $J^{\prime \prime}=0$ rotational state is used as threshold, i.e. when we essentially employ a laser frequency that 


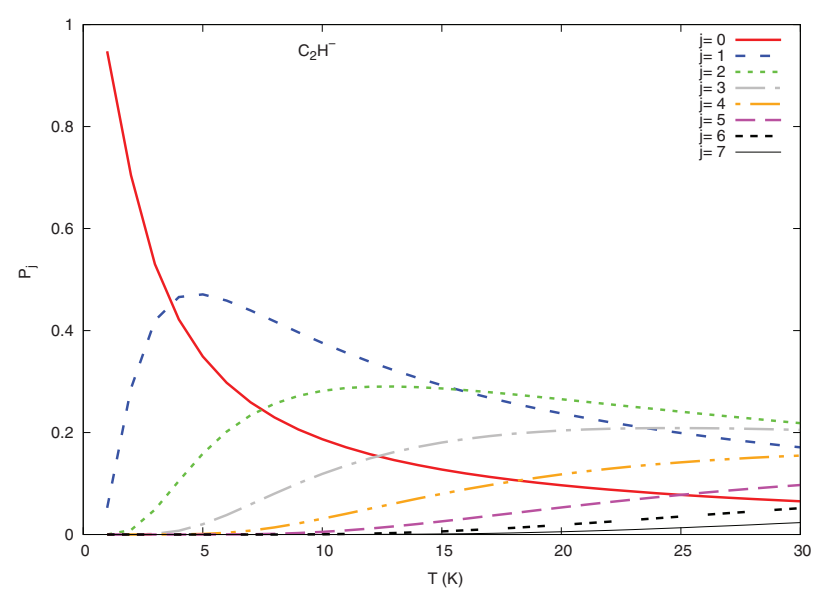

Fig. 6. Computed steady-state populations of the rotational levels of the title molecule over a range of temperatures similar to those expected in a cold ion trap experiment (adapted from Ref. [15]).

coincides with the experimental EA value, we obtain the same results shown by the centre panel of Figure 3 and the loss rates involve the dominant populations of the $J^{\prime \prime}=1$ and $J^{\prime \prime}=2$ levels, followed by similar but lower populations for the $J^{\prime \prime}=0$ and $J^{\prime \prime}=3$ levels. The $J^{\prime \prime}=4$ level then comes next in lower relative population. In other words, the "collision-dominated" regime applied during state preparation is also followed during the anion's decays as the laser is switched on.

As the selected $K_{J^{\prime \prime}}^{\mathrm{PD}}$ values are taken to exclude photodetaching the electron from anions in the $J^{\prime \prime}=0$ (centre panel), we see that the loss rates after switching the laser on change only very slightly their behaviour: the collisional re-populations of the anions in the $J^{\prime \prime}=1$ and $J^{\prime \prime}=2$ states is still active from the presence of the state-changing collisional rates involving the $J^{\prime \prime}=0$ and $J^{\prime \prime}=1$ levels which in turn are not depleted fast enough by the photo-detaching laser and therefore still are the more abundantly present in the trap. Once we move to the situation depicted by the right-side panel in the figure, we further exclude the $J^{\prime \prime}=1$ states from the photodetachment process. Hence, both the $J^{\prime \prime}=1$ and $J^{\prime \prime}=2$ states decay more slowly in comparison with the data in the left panel of that figure and the same effect occurs for the $J^{\prime \prime}=0, J^{\prime \prime}=3$ and the next higher states. In other words, to employ a frequency-selected laser allows one to change in the trap the relative interplay between collisional re-population and photon-induced population losses.

The data we report in Figure 5 extend the model by changing the laser frequency to start from different thresholds, thus excluding from the PD process different anion's states present in the trap after the thermalisation step. To better clarify this point, it may be useful to look first at the data reported by Figure 6, where we show the steady-state population of the anion's rotational states at temperatures around those discussed in the present study.

The data in that figure provide indications on the steady-state population in a trap depending on the selected temperature of the uploaded buffer gas. In the example chosen for the present analysis, our trap temperature is taken to be, in Figures 4 and 5, of $15 \mathrm{~K}$. From the data of Figure 6 we therefore see that levels $J=1,2$ and 3 have higher populations than the $J^{\prime \prime}=0$ state, with the $J^{\prime \prime}=4$ state just below in size. Since the collisional state-changing rates are largest for $\Delta J^{\prime \prime}=1$ and $\Delta J^{\prime \prime}=2$ transitions, after thermalisation in the trap and during the PD process (laser switched on) the re-populations by collisions involve dominantly all the states populated the most according the data of Figure 6 . Thus, when we prevent the PD process to involve the first three lower levels, as indicated in the left panel of Figure 5, we see that we now have an even larger fractional population of anions which are in their $J^{\prime \prime}=1$ and $J^{\prime \prime}=2$ states, and even anions in the $J^{\prime \prime}=3$ state are comparable in relative population to the $J^{\prime \prime}=0$ state. In other words, the selective PD process not involving the first two rotational states allows them to participate more efficiently to the collisional repopulation of the lower levels, thereby markedly delaying loss rates from those levels. This effect is even more clearly seen from the data reported in the next two panels of Figure 5. The panel in the centre of that figure shows our calculations when the laser frequency is just enough to photo-detach the extra electron from anion's molecules which are in their $J^{\prime \prime}=4$ rotational state. In other words, the $K_{J^{\prime \prime}}^{\mathrm{PD}}$ values are set to zero for PD processes from molecules in the lower rotational states: it means that repopulations between those levels and from those levels to the higher rotational states is only controlled by the collisional dynamics with the buffer gas. The molecular anions in the $J^{\prime \prime}=1$ and $J^{\prime \prime}=2$ states are leaving the trap much more slowly than before and their relative populations are also much larger. We also see that the selective role of collisional re-populations between states, driven by the different sizes of the state-to-state collisional rates in the trap (see earlier in this Section), is bringing up the residual population of the $J^{\prime \prime}=3$ state, which is now even larger than that of the $J^{\prime \prime}=0$ state.

The slowing down of the loss rates in the trap are also evident from the calculations presented in the right panel of the same Figure 5. The PD threshold of the laser has been now moved up to the $J^{\prime \prime}=5$ level. It means that all the lower five rotational states are not undergoing laserinduced losses while only the residual states from $J^{\prime \prime}=5$ to $J^{\prime \prime}=7$ are depopulated by the laser frequency switched on in the trap. We therefore see that the relative population losses in the trap are now less affected by the presence of the laser than in the other examples shown by previous figures: the more relatively populated rotational states remain those in which collisions have populated the $J^{\prime \prime}=1$ and $J^{\prime \prime}=2$ rotational states, which are now more abundant in the trap and decay more slowly than before. Their initial fractional populations during the PD time considered remain about twice that with the $J^{\prime \prime}=0$ state.

\section{Conclusions}

In the present work we have endeavoured to analyse the possible occurrence of state-population enhancements during PD processes in a cold ion trap under the 
presence of a buffer gas with which the molecular anions can modify by collisions the relative populations of their rotational states before the PD laser frequency is switched on. Thus, after the thermalisation step in which the trapped anions are brought to have their internal (i.e. rotational and translational) temperatures in line to that provided by the buffer He gas, we have analysed different trap parameters to see what effects they would have on the final anion's populations after photo-detaching the molecular anion's excess electron. We have therefore shown effects coming from changing the laser frequency from being exactly that of the experimentally known EA value for the molecular anion, to being well above that threshold. We have also modeled the PD process using Wigner's threshold law, we have shown that a laser frequency that matches the EA value causes slower loss rates and allows individual rotational states of the molecular anion to be kept longer in the trap.

We have also shown that increasing the buffer gas density does not affect on the PD step but does accelerate the thermalisation step of the relative rotational populations driven by the collision-induced repoplulation of those states. Such behaviour turned out to be particularly effective when the PD operating conditions were chosen to be in a "collision dominated" environment, i.e. without selecting a powerful laser source. When that condition is chosen instead, we have shown that one should expect the rate losses to be very fast and to be largely unaffected by the differences between state-changing collisional rates within the trap kinetics. The PD process therefore becomes essentially not state-selective. Under "collision dominated" choices of trapping parameters, we showed the effects of detaching the excess electron from a specific energy threshold associated with a selected rotational state. In other words, we have re-run the trap kinetics with laser frequencies less than the EA threshold value, thereby excluding photo-detaching electrons from molecular anions in rotational states lower than the one selected by the laser frequency. This study has indicated that the relative population decay rates of the $\mathrm{PD}$ excluded rotational states can be altered by modifying the operating conditions, thus producing different abundances of anions in different rotational states from those given by its prelaser switching situation.

The present calculations have shown that it would be possible, for molecular anions with a large number of rotational states collisionally populated in the trap, to remain significantly present in the cold trap after the laser-on step, and to selectively control the relative populations of some specific rotational states by an interplay between laser frequency/power changes and the collisional re-populations of the molecular states through the action of the buffer gas.

Such an analysis can obviously be extended to different systems and can help to guide the experimental choices of parameters during optimisation of $\mathrm{PD}$ parameters at the low temperatures of cold ion traps.

Open access funding provided by Austrian Science Fund (FWF). We are grateful to Malcolm Simpson for many useful discussion on their experimental treatment of PD processes.
All numerical data pertaining to our parametric fitting of the computed PES and to the actual values of the computed cross sections and state-changing rates are available on request from the authors. We further acknowledge the financial support of the Austrian Science Fund (FWF ) through research grant No. P29558-N36. One of us (L.G-S) further thanks MINECO (Spain) for grants CTQ2015-65033-P and PGD2018-096444-BI00.

\section{Author contribution statement}

All the authors were involved in the preparation of the manuscript. All the authors have read and approved the final manuscript. All authors contributed equally to the work.

Publisher's Note The EPJ Publishers remain neutral with regard to jurisdictional claims in published maps and institutional affiliations.

Open Access This is an open access article distributed under the terms of the Creative Commons Attribution License (https://creativecommons.org/licenses/by/4.0/), which permits unrestricted use, distribution, and reproduction in any medium, provided the original work is properly cited.

\section{References}

1. H. Ehrhardt, L.A. Morgan, Electron Collisions with Molecules, Clusters and Surfaces (Plenum, New York, 1994)

2. H. Massey, Negative Ions (Cambridge University Press, Cambridge, 1976)

3. A. Grill, Cold Plasmas in Materials Fabrication (IEEE Press, New York, 1994)

4. K.M. Ervin, W.C. Lineberger, Advances in Gas Phase Ion Chemistry (JAI, Greenwhich, 1992)

5. D. Offenberg, C. Wellers, C.B. Zhang, S. Schiller, J. Phys. B: At. Mol. Opt. Phys. 42, 035101 (2009)

6. R. Wester, J. Phys. B: At. Mol. Opt. Phys. 42, 154001 (2009)

7. D. Hauser, S. Lee, F. Carelli, S. Spieler, O. Lakhmanskaya, E.S. Endres, S.S. Kumar, F. Gianturco, R. Wester, Nat. Phys. 11, 467 (2015)

8. L. González-Sánchez, F.A. Gianturco, F. Carelli, R. Wester, New J. Phys. 17, 123003 (2015)

9. O. Lakhmanskaya, M. Simpson, S. Murauer, V. Kokoouline, R. Wester, J. Chem. Phys. 149, 104302 (2018)

10. E.P. Wigner, Phys. Rev. 73, 1002 (1948)

11. P. Lin, R.R. Lucchese, J. Chem. Phys. 114, 9350 (2001)

12. P.G. Wenthold, W.C. Linerberger, Acc. Chem. Res. 32, 597 (1999)

13. D.M. Neumark, Acc. Chem. Res. 26, 33 (1993)

14. F. Dumouchel, A. Spielfiedel, M. Senent, N. Feautrier, Chem. Phys. Lett. 533, 6 (2012)

15. F.A. Gianturco, L. González-Sánchez, B.P. Mant, R. Wester, J. Chem. Phys. 151, 144304 (2019)

16. X. Huang, T.J. Lee, J. Phys. Chem. 131, 104301 (2009)

17. U. Fano, R.P. Rau, Atomic Collisions and Spectra (Academic Press Inc, 1986) 
18. P.C. Engelking, Phys. Rev. A 26, 740 (1982)

19. J. Zhou, E. Gerand, D.M. Neumark, J. Chem. Phys. 127, 114313 (2007)

20. M. Simpson, M. Nötzold, T. Michaelsen, B. Bastian, J. Meyer, R. Wild, F.A. Gianturco, M. Milovanovic, V. Kokoouline, R. Wester, In preparation, 2020

21. F.A. Gianturco, O.Y. Lakhmanskaya, M. Hernández Vera, R. Yurtsever, R. Wester, Faraday Discuss. 212, 117 (2018)
22. S. Brünken, C.A. Gottlieb, H. Gupta, M.C. McCarthy, P. Thaddeus, Astron. Astrophys. 464, L33 (2007)

23. R.J. Saykally, L. Veseth, K.M. Evenson, J. Chem. Phys. 80, 2247 (1984)

24. D. López-Durán, E. Bodo, F.A. Gianturco, Comput. Phys. Commun. 179, 821 (2008)

25. M. Hernández Vera, F.A. Gianturco, R. Wester, H. da Silva, Jr., O. Dulieu, S. Schiller, J. Chem. Phys. 146, 124310 (2017) 